\title{
BMJ Open Socioeconomic status in relation to cardiovascular disease and cause- specific mortality: a comparison of Asian and Australasian populations in a pooled analysis
}

Mark Woodward, ${ }^{1,2,3}$ Sanne A E Peters, ${ }^{1,4}$ G David Batty, ${ }^{5,6,7}$ Hirotsugu Ueshima, ${ }^{8}$ Jean Woo, ${ }^{9}$ Graham G Giles, ${ }^{10}$ Federica Barzi, ${ }^{2}$ Suzanne C Ho, ${ }^{11}$ Rachel R Huxley, ${ }^{12}$ Hisatomi Arima, ${ }^{2,8}$ Xianghua Fang, ${ }^{13}$ Annette Dobson, ${ }^{12}$ Tai Hing Lam, ${ }^{14}$ Prin Vathesatogkit, ${ }^{2,15}$ on behalf of the Asia Pacific Cohort Studies Collaboration

To cite: Woodward M, Peters SAE, Batty GD, et al. Socioeconomic status in relation to cardiovascular disease and cause-specific mortality: a comparison of Asian and Australasian populations in a pooled analysis. BMJ Open 2015;5: e006408. doi:10.1136/ bmjopen-2014-006408

- Prepublication history and additional material is available. To view please visit the journal (http://dx.doi.org/ 10.1136/bmjopen-2014006408)

Received 22 August 2014 Revised 13 December 2014 Accepted 16 December 2014

CrossMark

For numbered affiliations see end of article.

Correspondence

Professor Mark Woodward;

mark.woodward@

georgeinstitute.ox.ac.uk

\section{ABSTRACT}

Objectives: In Western countries, lower socioeconomic status is associated with a higher risk of cardiovascular disease (CVD) and premature mortality. These associations may plausibly differ in Asian populations, but data are scarce and direct comparisons between the two regions are lacking. We, thus, aimed to compare such associations between Asian and Western populations in a large collaborative study, using the highest level of education attained as our measure of social status.

Setting: Cohort studies in general populations conducted in Asia or Australasia.

Participants: 303036 people (71\% from Asia) from 24 studies in the Asia Pacific Cohort Studies

Collaboration. Studies had to have a prospective cohort study design, have accumulated at least 5000 personyears of follow-up, recorded date of birth (or age), sex and blood pressure at baseline and date of, or age at, death during follow-up.

Outcome measures: We used Cox regression models to estimate relationships between educational attainment and CVD (fatal or non-fatal), as well as allcause, cardiovascular and cancer mortality.

Results: During more than two million person-years of follow-up, 11065 deaths (3655 from CVD and 4313 from cancer) and 1809 CVD non-fatal events were recorded. Adjusting for classical CVD risk factors and alcohol drinking, hazard ratios ( $95 \% \mathrm{Cls}$ ) for primary relative to tertiary education in Asia (Australasia) were $1.81(1.38,2.36)(1.10(0.99,1.22))$ for all-cause mortality, $2.47(1.47,4.17)(1.24(1.02,1.51))$ for CVD mortality, $1.66(1.00,2.78)(1.01(0.87,1.17))$ for cancer mortality and $2.09(1.34,3.26)(1.23(1.04$, 1.46)) for all CVD.

Conclusions: Lower educational attainment is associated with a higher risk of CVD or premature mortality in Asia, to a degree exceeding that in the Western populations of Australasia.

\section{Strengths and limitations of this study}

The large sample size and number of events, and the use of multiple imputation of missing values, controls for both random and systematic error, and thus enables accurate estimation of associations-although ideally there would be no missing values.

- The extensive and broad experience, both individually and collectively, of the authors ensures a cogent and informed synthesis of the results of this research project.

- The individual participant data were compiled from existing studies, without a common protocol.

- Education is a common measure of social status, but may be best regarded as a relative, than as an absolute, classification when comparing cultures.

\section{INTRODUCTION}

A large number of studies have clearly demonstrated higher rates of major causes of death-including cardiovascular disease (CVD), selected cancers, respiratory illness and injuries-in people who are more socially deprived. ${ }^{1-5}$ It is now well recognised that these associations are graded across the full spectrum of socioeconomic status (SES).

Most of this evidence is derived from studies of Western societies. In Asia, where the burden of chronic disease is increasing rapidly, ${ }^{6-8}$ there are prima facie reasons to anticipate that SES may have different relationships with risk to those seen for Western societies-body frames and environmental exposures, both of which are related to SES, 
would be expected to differ. Nevertheless, our recent review of SES and the risk of premature mortality in Asia $^{9}$ found an overall inverse association between SES and all-cause, CVD and cancer mortality, albeit with substantial between-study heterogeneity in the measures of SES used and effect sizes.

To our knowledge, a direct comparison of the effects of the same measure of SES between Oriental and Occidental populations has not previously been made. Data from the Asia Pacific Cohort Studies Collaboration (APCSC), a large scale individual data pooling project based in Asia and Australasia (Australia and New Zealand), affords us the opportunity to make such a comparison.

\section{METHODS}

\section{Study design}

Details of the APCSC have been described elsewhere. ${ }^{10}$ In brief, a study was eligible for inclusion if it met the following criteria: (1) the population was drawn from the Asia Pacific region; (2) it had a prospective cohort study design; (3) it had accumulated at least 5000 person-years of follow-up; (4) date of birth (or age), sex and blood pressure were recorded at baseline; (5) date of, or age at death was recorded during follow-up.

The index of SES used in this study was educational attainment, which was recorded in 24 studies within APCSC. This was self-reported and categorised into three groups: no completed education or completed only primary school; completed secondary school; and completed tertiary (university or college). Within each study, height and weight were ascertained by direct measurement; body mass index (BMI) was computed as weight $(\mathrm{kg}) /$ height $\left(\mathrm{m}^{2}\right)$. Blood pressure and total blood cholesterol were generally measured using standard protocols. ${ }^{10}$ Study participants provided information on cigarette smoking (current smoker/non-smoker) and alcohol drinking (yes/no). Cohorts were classified as Asian if the participants were recruited from mainland China, Hong Kong, Japan, Singapore, Taiwan or Thailand; and as Australasian if from Australia or New Zealand.

\section{End points}

All studies recorded deaths ${ }^{11}$ and eight studies additionally reported non-fatal stroke, and seven reported nonfatal coronary heart disease (CHD) outcomes. Cardiovascular events were defined as fatal or non-fatal CVD, CHD and stroke; non-fatal outcomes were not recorded for cancer.

\section{Statistical analysis}

We analysed the association between education and classical risk factors for chronic disease using $\chi^{2}$ tests for trend. The effects of SES on clinical outcomes were analysed using Cox regression models, stratified by sex and study, and the primary analyses were stratified by region (Asia/Australasia). Further, a priori, we planned to analyse the effects of SES adjusted for age and for a range of additional potential confounding factors: BMI, smoking and alcohol consumption, systolic blood pressure (SBP), blood cholesterol and diabetes. The effects of education were summarised by tests for trend across education groups by fitting education as an ordinal variable in the Cox models. Effect modification according to region was examined using Wald tests. In secondary analyses, the hazard ratios for primary or below $v$ tertiary education for all-cause mortality and CVD were explored within age groups by region, sex by region and across country-specific subgroups.

Many of our participants had missing values (see online supplementary appendix table S1) for several of the studied confounders (except age and SBP), with some confounders being missing for all individuals within particular studies. A variety of approaches are commonly used to deal with missing data. The complete case analysis-omitting participants with any missing value-is the simplest way which may result in reduced power and has potential for bias in the resulting estimates. Multiple imputation is a principled alternative, that can often improve power and reduce bias. Since some of the variables being imputed are not normally distributed, we imputed using the MICE algorithm, ${ }^{12}$ with (for computational advantage) 'study' taken as a fixed effect. Our imputation model also included educational attainment (primary or none/secondary/tertiary), age, sex, BMI, smoking status, alcohol status, SBP, blood cholesterol, diabetes, event status (yes/no), and days to event or censoring. Imputations were obtained by running independent chains of 1000 iterations to generate five imputed data sets. Sensitivity analyses included using a random effect for study in the imputation model (ie, a random intercept accounting for heterogeneity in the underlying baseline hazards across studies), multiple imputation using data augmentation, varying the random seed in the imputation process and the number of imputed data sets derived (all of which gave similar estimates; results not shown) and a complete case analysis (shown in the online supplementary appendix).

A p value $<0.05$ was considered significant; a priori, no corrections were made for multiple comparisons. Analyses were performed using R V.2.15.3 (R Foundation for Statistical Computing, Vienna, Austria) and SAS V.9.3 (SAS Institute Inc, Cary, North Carolina, USA).

\section{RESULTS}

The mean age of participants in the 24 cohorts combined was 47 years; $32 \%$ were female (table 1). Compared with participants from Asian cohorts, those from Australasia were generally older and more were female. As the level of educational attainment increased, the percentage of women decreased in both regions, but more especially in Asia (table 2). In Australasia, those in the higher education groups were younger and slightly leaner; these effects were incremental across the education groups. 
Table 1 Summary characteristics of participants in the 24 APCSC studies

\begin{tabular}{|c|c|c|c|c|c|c|c|c|}
\hline & $\mathbf{n}$ & $\begin{array}{l}\text { Baseline } \\
\text { year (19') } \\
\text { (range) }\end{array}$ & $\begin{array}{l}\text { Median } \\
\text { follow-up } \\
\text { (years) }\end{array}$ & $\begin{array}{l}\text { Female } \\
(\%)\end{array}$ & $\begin{array}{l}\text { Mean age } \\
\text { (years) } \\
\text { (SD) }\end{array}$ & $\begin{array}{l}\text { Total } \\
\text { deaths }\end{array}$ & $\begin{array}{l}\text { Total } \\
\text { CVD } \\
\text { events }\end{array}$ & $\begin{array}{l}\text { Total } \\
\text { cancer } \\
\text { deaths }\end{array}$ \\
\hline Australasia & 86835 & 78-99 & 8.1 & 45 & $54(14)$ & 5334 & 2323 & 2254 \\
\hline ALSA & 567 & $92-93$ & 4.7 & 35 & $78(6)$ & 167 & 72 & 22 \\
\hline ANHF & 9277 & $89-90$ & 8.3 & 51 & $43(13)$ & 374 & 115 & 154 \\
\hline Canberra & 834 & $90-91$ & 9.7 & 45 & $77(5)$ & 552 & 211 & 100 \\
\hline Fletcher Challenge & 10298 & $92-94$ & 5.8 & 28 & $44(15)$ & 372 & 465 & 135 \\
\hline Melbourne & 41286 & $90-94$ & 8.5 & 59 & $55(9)$ & 2081 & 551 & 1112 \\
\hline Newcastle & 5933 & 83-94 & 8.9 & 50 & $52(10)$ & 516 & 204 & 215 \\
\hline Perth & 6444 & $78-94$ & 14.4 & 51 & 45 (13) & 299 & 103 & 117 \\
\hline WA AAA Screenees & 12196 & $96-99$ & 3.2 & 0 & $72(4)$ & 973 & 602 & 399 \\
\hline Asia & 216201 & $77-97$ & 7.1 & 27 & $44(10)$ & 5731 & 2106 & 2059 \\
\hline Akabane (Japan) & 1806 & $85-86$ & 11.0 & 55 & $54(8)$ & 133 & 77 & 55 \\
\hline Anzhen (China) & 8378 & 91 & 4.3 & 55 & $54(13)$ & 322 & 273 & 66 \\
\hline Anzhen 02 (China) & 4152 & 92 & 3 & 51 & $47(8)$ & 19 & 17 & 0 \\
\hline Beijing Aging (China) & 2092 & 92 & 4.8 & 51 & $70(9)$ & 428 & 204 & 48 \\
\hline CISCH (China) & 2167 & $92-93$ & 3.3 & 51 & $44(7)$ & 7 & 23 & 3 \\
\hline CVDFACTS (Taiwan) & 5729 & $88-96$ & 6.0 & 55 & $47(15)$ & 228 & 60 & 65 \\
\hline East Beijing (China) & 1102 & $77-94$ & 17.1 & 51 & $44(15)$ & 109 & 60 & 19 \\
\hline EGAT (Thailand) & 3499 & 85 & 11.4 & 23 & $43(5)$ & 165 & 51 & 43 \\
\hline Fangshan (China) & 2602 & $91-92$ & 3.6 & 66 & $47(10)$ & 48 & 51 & 8 \\
\hline Guangzhou Occupational (China) & 166282 & $85-97$ & 7.1 & 22 & $41(6)$ & 2398 & 568 & 1197 \\
\hline Hong Kong (Hong Kong) & 2973 & $85-91$ & 2.5 & 57 & $79(7)$ & 652 & 202 & 127 \\
\hline Kinmen (China) & 2783 & 93-96 & 2.9 & 48 & $63(10)$ & 207 & 118 & 54 \\
\hline Miyama (Japan) & 1055 & $88-90$ & 6.6 & 56 & $61(10)$ & 88 & 22 & 36 \\
\hline Singapore NHS92 (Singapore) & 3305 & 92 & 6.2 & 52 & $39(12)$ & 71 & 78 & 22 \\
\hline Xi'an (China) & 1695 & 76 & 19.7 & 34 & $44(6)$ & 225 & 80 & 77 \\
\hline Yunnan (China) & 6581 & 92 & 4.5 & 3 & $56(9)$ & 631 & 222 & 239 \\
\hline Overall & 303036 & 77-99 & 7.3 & 32 & $47(12)$ & 11065 & 4429 & 4313 \\
\hline
\end{tabular}

Bold typeface indicates the overall baseline characteristics for the two regions, and for the two regions combined.

ALSA, Australian Longitudinal Study of Aging; ANHF, Australian National Heart Foundation; APCSC, Asia- Pacific Cohort Studies

Collaboration; CISCH, Capital Iron and Steel Company Hospital; CVD, cardiovascular disease (comprise of fatal and non-fatal events);

CVDFACTS, Cardiovascular Disease Risk Factors Two-Township Study; EGAT, Electricity Generating Authority of Thailand Study; NHS92,

National Health Study 1992; WA AAA Screenees, Western Australian Abdominal Aortic Aneurysm Screenees.

Australasian men and women who received tertiary education were more likely to be alcohol drinkers, but less likely to smoke or have diabetes, and had lower levels of blood pressure and cholesterol than others. In the Asian studies, the gradient between education and diabetes was weaker and the gradients for alcohol drinking and mean cholesterol were reversed.

Over a median of 7.3 years of follow-up, 11065 deaths were recorded, of which 3655 were due to GVD and 4313 were cancer (table 3). Both adjusting for the effects of age and sex and additionally adjusting for several confounders, participants with the highest educational attainment had the lowest risk of all-cause mortality, and those with the lowest attainment had the highest risk. While this gradient was seen in Asian and Australasian studies, it was markedly steeper in Asia ( $p$ value for interaction $<0.0001$ ). After full adjustment, in Asia, those with primary education or below had a $56 \%$ higher risk of death during follow-up, compared with $14 \%$ in Australasia. CVD mortality was also inversely associated with education in Asian and Australasia populations; again, the association was strongest in Asia $(\mathrm{p}=0.0002)$. An inverse association with increasing level of educational attainment was also found for death from cancer in the Asian studies, although the weak gradient was not statistically significant in Australasia. Education was significantly and inversely associated with other causes of death (non-CVD or cancer) in both regions: more strongly so $(p=0.04)$, in Asia. Adjusting for potential confounders only partially attenuated the association between education and all outcomes in both regions.

Table 4 shows the inverse associations of education with all CVD, CHD and stroke events, including nonfatal outcomes in each case. Results for all CVD were similar to those for CVD mortality. For all CHD and all stroke, inverse gradients with educational attainment were apparent, consistent with all other outcomes but the hazard ratios for CHD were relatively weak and virtually identical in the two regions $(\mathrm{p}=0.80$, after full adjustment). For stroke, the hazard ratios were more extreme in Asia ( $p=0.007)$; Asians with the lowest educational attainment had a $54 \%$ higher risk of stroke, compared with the highest attainment, after full adjustment; the corresponding estimate for Australasia was a non- 
Table 2 Baseline age-adjusted and sex-adjusted mean value (or percentage, where stated) by level of education attained

\begin{tabular}{|c|c|c|c|}
\hline & \multicolumn{3}{|c|}{ Educational attainment } \\
\hline & Tertiary & Secondary & Primary or none \\
\hline \multicolumn{4}{|l|}{ Australasia } \\
\hline $\mathrm{n}$ & 20955 & 24190 & 41690 \\
\hline Age (year) & 56 & 58 & 61 \\
\hline Female (\%) & 41 & 44 & 54 \\
\hline BMI $\left(\mathrm{kg} / \mathrm{m}^{2}\right)$ & 25.3 & 26.0 & 26.9 \\
\hline Systolic blood pressure $(\mathrm{mm} \mathrm{Hg})$ & 132 & 135 & 136 \\
\hline Total blood cholesterol (mmol/L) & 5.47 & 5.57 & 5.63 \\
\hline Cigarette smokers (\%) & 10 & 19 & 22 \\
\hline Alcohol drinkers (\%)* & 86 & 82 & 73 \\
\hline Diabetes (\%) & 2.1 & 3.3 & 4.4 \\
\hline \multicolumn{4}{|l|}{ Asia } \\
\hline $\mathrm{n}$ & 25774 & 132853 & 57574 \\
\hline Age (year) & 50 & 48 & 55 \\
\hline Female $(\%)$ & 30 & 39 & 60 \\
\hline Body mass index $\left(\mathrm{kg} / \mathrm{m}^{2}\right)$ & 22.7 & 22.9 & 23.3 \\
\hline Systolic blood pressure $(\mathrm{mm} \mathrm{Hg})$ & 123 & 124 & 124 \\
\hline Total blood cholesterol (mmol/L) & 4.89 & 4.84 & 4.79 \\
\hline Cigarette smokers (\%) & 16 & 30 & 42 \\
\hline Alcohol drinkers (\%)* & 16 & 20 & 33 \\
\hline Diabetes $(\%) \dagger$ & 4.3 & 4.6 & 4.7 \\
\hline
\end{tabular}

*Excludes Canberra, Anzhen 02 and Xian studies, where information on alcohol drinking was not collected.

All tests for trend have $p<0.0001$ except $\dagger p=0.25$.

Table 3 Hazard ratios $(95 \% \mathrm{Cl})$ for level of education attained in relation to major causes of death

\begin{tabular}{|c|c|c|c|c|c|}
\hline \multirow[b]{2}{*}{ Adjustment } & \multicolumn{3}{|c|}{ Educational attainment } & \multirow{2}{*}{$\begin{array}{l}p \text { Value } \\
\text { for trend }\end{array}$} & \multirow{2}{*}{$\begin{array}{l}\mathrm{p} \text { Value for } \\
\text { interaction by region }\end{array}$} \\
\hline & Tertiary & Secondary & Primary or none & & \\
\hline \multicolumn{6}{|c|}{ All-cause mortality } \\
\hline \multicolumn{6}{|c|}{ Australasia } \\
\hline Age, sex & 1 & 1.11 (1.02 to 1.21$)$ & 1.19 (1.10 to 1.29$)$ & $<0.001$ & \\
\hline Multiple & 1 & 1.08 (0.99 to 1.18$)$ & 1.14 (1.05 to 1.23$)$ & 0.001 & \\
\hline \multicolumn{6}{|l|}{ Asia } \\
\hline Age, sex & 1 & 1.26 (1.11 to 1.42$)$ & 1.64 (1.46 to 1.85$)$ & $<0.001$ & $<0.001$ \\
\hline Multiple & 1 & 1.21 (1.07 to 1.36$)$ & 1.56 (1.38 to 1.76$)$ & $<0.001$ & $<0.001$ \\
\hline \multicolumn{6}{|c|}{ Cardiovascular mortality } \\
\hline \multicolumn{6}{|c|}{ Australasia } \\
\hline Age, sex & 1 & $1.16(1.00$ to 1.35$)$ & 1.31 (1.15 to 1.51$)$ & $<0.001$ & \\
\hline Multiple & 1 & $1.10(0.94$ to 1.28$)$ & 1.20 (1.04 to 1.38$)$ & 0.01 & \\
\hline \multicolumn{6}{|l|}{ Asia } \\
\hline Age, sex & 1 & $1.30(1.03$ to 1.64$)$ & 1.89 (1.51 to 2.37$)$ & $<0.001$ & 0.0008 \\
\hline Multiple & 1 & $1.23(0.98$ to 1.56$)$ & 1.78 (1.42 to 2.23$)$ & $<0.001$ & 0.0002 \\
\hline \multicolumn{6}{|c|}{ Cancer mortality } \\
\hline \multicolumn{6}{|c|}{ Australasia } \\
\hline Age, sex & 1 & $1.05(0.92$ to 1.20$)$ & 1.09 (0.97 to 1.23$)$ & 0.12 & \\
\hline Multiple & 1 & $1.03(0.90$ to 1.18$)$ & 1.07 (0.95 to 1.20$)$ & 0.27 & \\
\hline \multicolumn{6}{|l|}{ Asia } \\
\hline Age, sex & 1 & 1.21 (1.01 to 1.45$)$ & $1.52(1.26$ to 1.84$)$ & $<0.001$ & 0.001 \\
\hline Multiple & 1 & $1.16(0.96$ to 1.39$)$ & 1.39 (1.15 to 1.69$)$ & $<0.001$ & 0.01 \\
\hline \multicolumn{6}{|c|}{ Mortality due to other causes (not cardiovascular, not cancer) } \\
\hline \multicolumn{6}{|c|}{ Australasia } \\
\hline Age, sex & 1 & 1.16 (0.98 to 1.38$)$ & 1.24 (1.06 to 1.45$)$ & 0.01 & \\
\hline Multiple & 1 & 1.16 (0.98 to 1.39$)$ & 1.23 (1.04 to 1.46$)$ & 0.02 & \\
\hline \multicolumn{6}{|l|}{ Asia } \\
\hline Age, sex & 1 & $1.24(1.00$ to 1.53$)$ & 1.58 (1.28 to 1.96$)$ & $<0.001$ & 0.04 \\
\hline Multiple & 1 & $1.21(0.98$ to 1.50$)$ & 1.56 (1.26 to 1.94$)$ & $<0.001$ & 0.04 \\
\hline
\end{tabular}

Multiple adjustment is for age, sex, systolic blood pressure, total cholesterol, body mass index, smoking and alcohol drinking. For numbers of events, see online supplementary appendix table S4. 
Table 4 Hazard ratios $(95 \% \mathrm{Cl})$ for level of education attained in relation to cardiovascular disease

\section{Educational attainment}

\begin{tabular}{|c|c|c|c|c|c|}
\hline \multirow[b]{2}{*}{ Adjustment } & \multicolumn{3}{|c|}{ Educational attainment } & \multirow{2}{*}{$\begin{array}{l}\text { p Value } \\
\text { for linearity }\end{array}$} & \multirow{2}{*}{$\begin{array}{l}\text { p Value for } \\
\text { interaction } \\
\text { by region }\end{array}$} \\
\hline & Tertiary & Secondary & Primary or none & & \\
\hline \multicolumn{6}{|c|}{ All cardiovascular disease (fatal or non-fatal) } \\
\hline \multicolumn{6}{|c|}{ Australasia } \\
\hline Age, sex & 1 & 1.12 (0.99 to 1.27$)$ & $1.23(1.10$ to 1.39$)$ & $<0.001$ & \\
\hline Multiple & 1 & 1.04 (0.92 to 1.18$)$ & $1.11(0.99$ to 1.25$)$ & 0.0693 & \\
\hline \multicolumn{6}{|l|}{ Asia } \\
\hline Age, sex & 1 & 1.15 (0.94 to 1.42$)$ & $1.73(1.42$ to 2.11$)$ & $<0.001$ & $<0.001$ \\
\hline Multiple & 1 & 1.08 (0.88 to 1.33$)$ & 1.61 (1.32 to 1.96$)$ & $<0.001$ & $<0.001$ \\
\hline \multicolumn{6}{|c|}{ All coronary disease (fatal or non-fatal) } \\
\hline \multicolumn{6}{|c|}{ Australasia } \\
\hline Age, sex & 1 & 1.18 (1.00 to 1.39$)$ & 1.33 (1.14 to 1.56$)$ & $<0.001$ & \\
\hline Multiple & 1 & 1.05 (0.89 to 1.23$)$ & 1.15 (0.98 to 1.35$)$ & 0.06 & \\
\hline \multicolumn{6}{|l|}{ Asia } \\
\hline Age, sex & 1 & $1.10(0.77$ to 1.56$)$ & $1.32(0.93$ to 1.86$)$ & 0.08 & 0.95 \\
\hline Multiple & 1 & 1.01 (0.71 to 1.44$)$ & 1.18 (0.83 to 1.67$)$ & 0.25 & 0.80 \\
\hline \multicolumn{6}{|c|}{ All stroke (fatal or non-fatal) } \\
\hline \multicolumn{6}{|c|}{ Australasia } \\
\hline Age, sex & 1 & $1.12(0.89$ to 1.41$)$ & $1.17(0.94$ to 1.47$)$ & 0.18 & \\
\hline Multiple & 1 & 1.06 (0.84 to 1.33$)$ & 1.08 (0.86 to 1.36$)$ & 0.52 & \\
\hline \multicolumn{6}{|l|}{ Asia } \\
\hline Age, sex & 1 & $1.08(0.80$ to 1.45$)$ & $1.72(1.30$ to 2.26$)$ & $<0.001$ & 0.01 \\
\hline Multiple & 1 & 1.01 (0.75 to 1.36$)$ & 1.54 (1.17 to 2.04$)$ & $<0.001$ & 0.01 \\
\hline
\end{tabular}

Multiple adjustment is for age, sex, systolic blood pressure, total cholesterol, body mass index, smoking and alcohol drinking.

For numbers of events, see online supplementary appendix table S5.

significant $8 \%$. In these Asian data the stroke to CHD event ratio was roughly 2:1, whereas in Australasia it was about 1:2. Consequently the hazard ratios for stroke and CVD are relatively similar in Asia whereas the hazard ratios for CHD and CVD are relatively similar in Australasia.

There was no evidence that the associations between education and either all-cause mortality or total CVD differed by age or sex in either region (figure 1). Country-specific analyses suggested that the differential between low and high educational attainment, in the risks of all-cause mortality and all CVD, was largest in China, Taiwan and Thailand (figure 2). Results for Singapore were unreliable due to small numbers, but for the other Asian countries results were similar to those from Australasia.

Results from the complete case analysis were broadly similar, especially in Australasia where there were relatively few missing values (see online supplementary appendix tables S2-S5). In Asia, the hazard ratios for primary or no education versus tertiary education were generally higher in the complete case analysis, but not so much as to alter the conclusions drawn (see online supplementary appendix tables S4 and S5).

\section{DISCUSSION}

As far as we are aware, this is the first study to have examined the relationship between SES and mortality experience simultaneously in participants living in Asia and Western societies. We found that a relatively high educational attainment was associated with a lower risk of mortality and CVD in both populations. Overall, the gradients tended to be steeper in Asia than Australasia, with the greatest differential between low and high educational attainment being mostly seen in the least welldeveloped countries that were included in our study: China, Taiwan and Thailand.

\section{Education as a measure of SES}

The highest level of education attained has clear influence on occupational opportunities and earning potential. It is an attractive measure of SES for international comparisons because it is likely to be relatively well standardised between countries and is easily obtained from a standard questionnaire. ${ }^{13}$ Other advantages, compared with measures based on income or occupation, is that educational attainment is specific to an individual, relevant after retirement and not subject to possible reverse causality, whereby illness causes loss of income or employment, preceding death or clinical diagnosis of disease. On the other hand, educational attainment will rarely capture knowledge and experience gained through life, while economic returns, for the same level of education, may differ significantly across ethnic and sex groups. In our review of SES measures in Asia, educational attainment was a stronger predictor of in-study mortality than either income or occupation. ${ }^{9}$ 
Hazard ratio

$(95 \% \mathrm{Cl})$

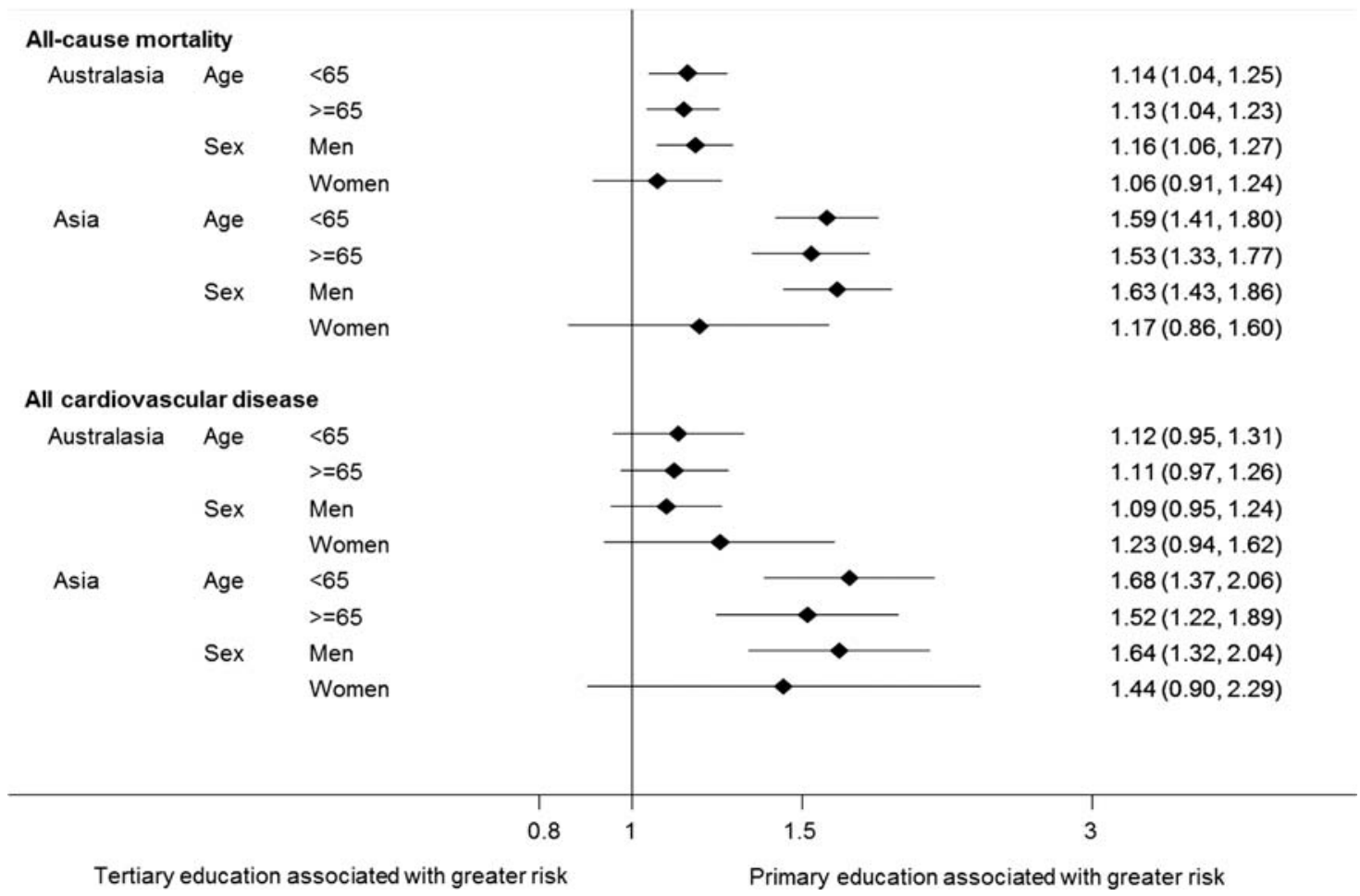

Figure 1 Hazard ratios $(95 \% \mathrm{Cls})$ for primary or below versus tertiary education in relation to all-cause mortality and total cardiovascular disease, by age within region and sex within region. Hazard ratios are adjusted for age and sex (as appropriate) plus systolic blood pressure, total cholesterol, body mass index, smoking and alcohol drinking.

\section{Education and risk profiles in the Asia-Pacific region}

Our results show that lower educational attainment was associated with less favourable risk profiles in Asia and Australasia. As in previous studies, ${ }^{2} 1415$ we found inverse relationships between education and age, SBP, smoking and diabetes, with the exception that the proportion of alcohol drinkers in studies from Australasia was higher in the best educated group-consistent with previous studies in the West. ${ }^{16-18}$ Mean BMI has, as in this study, often been found to be positively related to social deprivation, although not inevitably. ${ }^{19}{ }^{20}$ The association between serum cholesterol and level of education found in Asian and Australasian studies is consistent with previous literature which showed an inverse association between education and serum cholesterol in highincome countries but the reverse in low-income to middle-income countries. ${ }^{2} 2122$ These differing patterns reflect rapid urbanisation of the latter, in which more educated people are more likely to move to urban areas and adopt less healthy lifestyles, such as sedentary behaviour and the consumption of proatherogenic diets. ${ }^{23}$

\section{Education and non-communicable disease mortality} in the Asia-Pacific region

Although major risk factors such as diabetes, high-blood pressure and smoking contributed to the mortality and morbidity in both regions, we generally found a steeper gradient of the effect of education on adverse outcomes in Asia compared with Australasia. This might be explained by differences in national social and economic policies. In a study of the relationship between education and mortality in nine industrialised countries, Kunst $e t a l^{24}$ found that inequalities in mortality were twice as large in the USA, Italy and France as in the Netherlands, Denmark, Sweden and Norway and proposed that one potentially relevant factor was welfare and income policies. During the 1970s, income inequalities were relatively large in the USA, France and Italy and relatively small in the other countries. In our study, the majority of participants from Asia were living in China where the inequality gap was large in the 1990s. ${ }^{25}$ Thailand and Singapore were also in a period of rapid economic transformation during that period.

Healthcare policy and infrastructure may also play an important role. In Australia and New Zealand, like most other high-income countries, medical resources are relatively plentiful, more equitable and accessible than in China. While the healthcare reform in Australia has been well-organised since 1975, China introduced healthcare reform as late as 1997. This reform has exacerbated inequalities. ${ }^{26}{ }^{27}$ Many public hospitals and healthcare centres in China had low government subsidies and had to rely on increasing charges from patients. Profitable hospitals were increasingly built by private entrepreneurs. As a consequence, the lack of government funding for accessible and affordable healthcare 
Hazard ratio

$(95 \% \mathrm{Cl})$

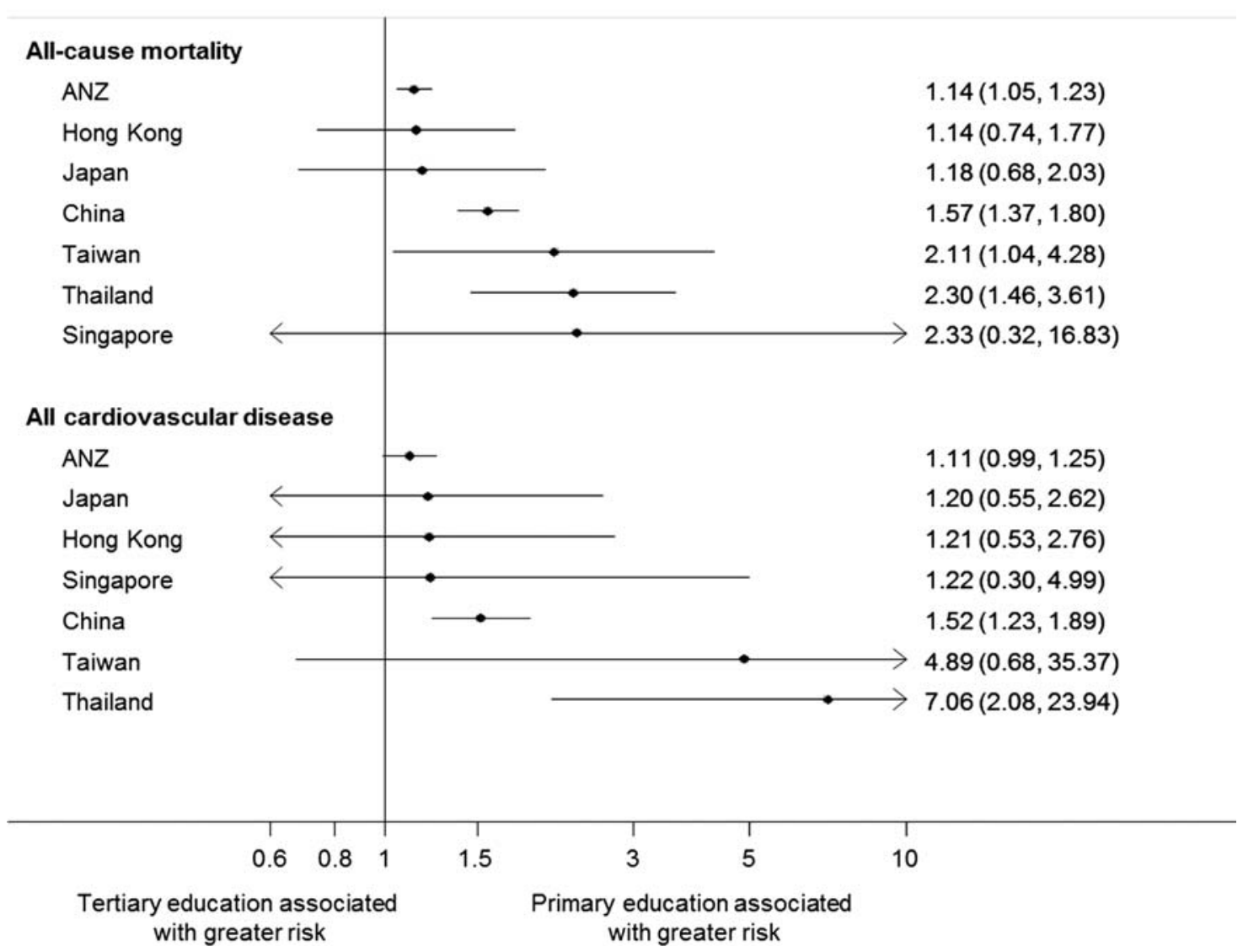

Figure 2 Hazard ratios (95\% Cls) for primary or below versus tertiary education in relation to all-cause mortality and total cardiovascular disease, by country. Hazard ratios are for age, sex, systolic blood pressure, total cholesterol, body mass index, smoking and alcohol drinking. ANZ, Australia and New Zealand.

coverage became a primary cause of socioeconomic disparity in health as medical resources were concentrated in urban hospitals and the individual out-of-pocket expenses for health expenditures increased sharply. ${ }^{27}$

Differential effects of educational attainment on health outcomes could also be influenced by personal, household and neighbourhood factors. ${ }^{3}{ }^{28}$ For example, variations in an individual's intelligence, religious belief and stress coping mechanism could intervene in the effect of education on health. A study from Scotland has shown that, after adjustment for IQ the risk gradient across five socioeconomic indicators was markedly attenuated for both coronary heart disease and all-cause mortality. ${ }^{29}$ The family structure can be a major cause of health disparities in the elderly (different support from different family backgrounds, family size or education of the offspring). This is more likely to be an issue in Asia than Australasia; for instance, the percentage of the elderly living in nursing homes in China is less than half that in Australia. ${ }^{30}{ }^{31}$ Environmental impoverishment, as depicted by area SES, has also been linked to health status and mortality. ${ }^{32} 33$
In general, those with better education are more likely to be aware of cancer symptoms, to have the opportunity for cancer screening, and to have more advanced and effective treatment. These factors probably explain the clear gradients with SES in our Asian studies. The lack of such effects in our Australasian studies is likely to be due to more homogeneous access to healthcare. There may also be differential effects by type of cancer. For example, level of education has been found to be inversely related to lung cancer, due to a lower prevalence of smoking among more highly educated people. ${ }^{34}{ }^{35}$ In contrast, breast cancer risk is known to be greater for the more highly educated, presumably because of different reproductive patterns, such as delayed childbearing and fewer children. ${ }^{36}$ Colon and prostate cancer incidence have been inconsistently associated with level of education. ${ }^{37}$ In our study, cancer deaths were too few (especially in Asia) to reliably investigate each cancer individually, although age-adjusted hazard ratios for the most common cancers by region and sex are shown in online supplementary appendix table S6. Except for ovarian cancer in Asia, the crude 
death rate for the group with only primary education was always the highest.

\section{Strengths and limitations}

Our study has the strength of a large sample size, which has allowed us to produce reliable estimates, at subgroup levels, for several key outcomes. We have only considered educational attainment as a measure of SES, but we would expect broadly similar results should another measure have been used, based on our past experience with contrasting measures of SES in Asian and Western populations. ${ }^{9} 38$

One limitation with our Collaboration is that details on how events, especially non-fatal events, were captured are lacking. In this specific study, the biggest limitation is the great number of missing values for cholesterol, diabetes and BMI, especially in the Asian studies (see online supplementary appendix table S1). Our previous analyses ${ }^{39}$ suggested it was unlikely that multiple imputation would improve precision compared with a complete case analysis, because of the high degree of missingness, but we would expect it to lead to reduced bias. Consequently we have chosen to report values from multiple imputation as our primary results. Most missing values in the Asian data were from a Chinese occupational cohort, which contributed $77 \%$ of the data. By removing these subjects, the 'healthy worker effect' has been reduced. In Australasia, the complete case analysis resulted in the removal of participants from the two studies with the highest average age, reducing the mean age of the participants from 54 to 51 years. Conceivably, these systematic differences could have contributed to the general attenuation of hazard ratios with the complete case analysis, although our subgroup analyses did not suggest heterogeneity of the effects of education by age. Overall, the main conclusions from our study are the same whether or not we account for missingness. Another limitation was that the large Chinese occupational study dominates our Asian data, which limits our ability to make generalisations across Asia. Finally, those classified as primary or below in Asia were likely to have included more with no schooling at all than in Australasia, whereas those with the highest education in Australasia were likely to have more with postgraduate education. This could have exaggerated the difference in the observed gradients between the two regions.

\section{CONCLUSIONS}

Our study underscores the importance of disparities in educational attainment in cardiovascular disease, cancer and other causes of death in Asia and Australasia and, hence, the crucial role of education in disease prevention. The greater magnitude of the effect in Asia, and the huge number of people living there, suggests that national policies to strengthen education within the region would have an immense positive impact on human health.
Author affiliations

${ }^{1}$ Nuffield Department of Population Health, The George Institute for Global Health, University of Oxford, Oxford, UK

${ }^{2}$ The George Institute for Global Health, University of Sydney, Sydney, Australia

${ }^{3}$ Department of Epidemiology, Johns Hopkins Bloomberg School of Public Health, Johns Hopkins University, Baltimore, USA

${ }^{4}$ Julius Center for Health Sciences and Primary Care, University Medical

Center Utrecht, Utrecht, The Netherlands

${ }^{5}$ Department of Epidemiology and Public Health, University College London, London, UK

${ }^{6}$ Centre for Cognitive Ageing and Cognitive Epidemiology, University of Edinburgh, Edinburgh, UK

${ }^{7}$ Alzheimer Scotland Dementia Research Centre, University of Edinburgh, Edinburgh, UK

${ }^{8}$ Department of Health Science, Shiga University of Medical Science, Shiga, Japan

${ }^{9}$ Faculty of Medicine, The Chinese University of Hong Kong, Hong Kong, China

${ }^{10}$ Cancer Epidemiology and Intelligence Division, Cancer Council Victoria, Melbourne, Australia

${ }^{11}$ Division of Epidemiology, School of Public Health and Primary Care, The Chinese University of Hong Kong, Hong Kong, China

${ }^{12}$ Faculty of Medicine and Biomedical Sciences, School of Population Health, University of Queensland, Australia

${ }^{13}$ Xuanwu Hospital, Capital Medical University, Bejing, China

${ }^{14}$ School of Public Health, The University of Hong Kong, Hong Kong, China

${ }^{15}$ Faculty of Medicine, Ramathibodi Hospital, Mahidol University, Thailand

Contributors Data analysis was performed by SAEP, FB and PV under the supervision of MW. HU, JW, GGG, SCH, XF, AD and THL supplied data.

The manuscript was drafted by MW, GDB and PV and commented on by all the other authors.

Funding This research received no specific grant from any funding agency in the public, commercial or not-for-profit sectors.

Competing interests None.

Ethics approval University of Sydney.

Provenance and peer review Not commissioned; externally peer reviewed.

Data sharing statement No additional data are available.

Open Access This is an Open Access article distributed in accordance with the Creative Commons Attribution Non Commercial (CC BY-NC 4.0) license, which permits others to distribute, remix, adapt, build upon this work noncommercially, and license their derivative works on different terms, provided the original work is properly cited and the use is non-commercial. See: http:// creativecommons.org/licenses/by-nc/4.0/

\section{REFERENCES}

1. Houweling TA, Kunst AE, Mackenbach JP. World Health Report 2000: inequality index and socioeconomic inequalities in mortality. Lancet 2001;357:1671-2.

2. Kaplan GA, Keil JE. Socioeconomic factors and cardiovascular disease: a review of the literature. Circulation 1993;88(4 Pt 1):1973-98.

3. Williams D, Collins C. US socioeconomic and racial differences in health: patterns and explanations. Annu Rev Soc 1995;21:349-86.

4. Adler NE, Boyce T, Chesney MA, et al. Socioeconomic status and health. The challenge of the gradient. Am Psychol 1994;49:15-24.

5. Feinstein JS. The relationship between socioeconomic status and health: a review of the literature. Milbank Q 1993;71:279-322.

6. Yoon KH, Lee JH, Kim JW, et al. Epidemic obesity and type 2 diabetes in Asia. Lancet 2006;368:1681-8.

7. World Health Organization. Global burden of disease: 2004 update. Geneva: World Health Organization, 2008.

8. World Health Organization. Global health risks: mortality and burden of disease attributable to selected major risks. Geneva: World Health Organization, 2009.

9. Vathesatogkit P, Batty GD, Woodward M. Socioeconomic disadvantage and disease-specific mortality in Asia: systematic review with meta-analysis of population-based cohort studies. $J$ Epidemiol Community Health 2014;68:375-83. 
10. Woodward M, Barzi F, Martiniuk A, et al. Cohort profile: the Asia Pacific Cohort Studies Collaboration. Int J Epidemiol 2006;35:1412-16.

11. International Classification of Diseases, Ninth Revision. Cdcgov National Center for Health Statistics, 2009 Web 22 November. 2013.

12. Resche-Rigon M, White IR, Bartlett JW, et al. Multiple imputation for handling systematically missing confounders in meta-analysis of individual participant data. Stat Med 2013;32:4890-905.

13. Mackenbach JP, Stirbu I, Roskam AJ, et al. Socioeconomic inequalities in health in 22 European countries. N Engl J Med 2008;358:2468-81.

14. Winkleby MA, Jatulis DE, Frank E, et al. Socioeconomic status and health: how education, income, and occupation contribute to risk factors for cardiovascular disease. Am J Public Health 1992;82:816-20.

15. Winkleby MA, Kraemer HC, Ahn DK, et al. Ethnic and socioeconomic differences in cardiovascular disease risk factors: findings for women from the Third National Health and Nutrition Examination Survey, 1988-1994. JAMA 1998;280:356-62.

16. Tomiak M, Gentleman JF, Jette M. Health and gender differences between middle and senior managers in the Canadian Public Service. Soc Sci Med 1997;45:1589-96

17. Emslie C, Hunt K, Maclntyre S. How similar are the smoking and drinking habits of men and women in non-manual jobs? Eur J Public Health 2002;12:22-8.

18. Marmot MG, North F, Feeney A, et al. Alcohol consumption and sickness absence: from the Whitehall II study. Addiction 1993;88:369-82.

19. Ball K, Crawford D. Socioeconomic status and weight change in adults: a review. Soc Sci Med 2005;60:1987-2010.

20. Silventoinen $\mathrm{K}$, Tatsuse T, Martikainen $\mathrm{P}$, et al. Occupational class differences in body mass index and weight gain in Japan and Finland. J Epidemiol 2013;23:443-50.

21. Garrison RJ, Gold RS, Wilson PW, et al. Educational attainment and coronary heart disease risk: the Framingham Offspring Study. Prev Med 1993;22:54-64.

22. Goyal A, Bhatt DL, Steg PG, et al. Attained educational level and incident atherothrombotic events in low- and middle-income compared with high-income countries. Circulation 2010;122:1167-75.

23. Yusuf S, Reddy S, Ounpuu S, et al. Global burden of cardiovascular diseases: part I: general considerations, the epidemiologic transition, risk factors, and impact of urbanization. Circulation 2001;104:2746-53.

24. Kunst AE, Mackenbach JP. The size of mortality differences associated with educational level in nine industrialized countries. $\mathrm{Am}$ J Public Health 1994;84:932-7.
25. Gini Index. World development indicators. World Bank, Development Research Group, 2011.

26. Palmer GR, Short SD. Health care \& public policy: an Australian analysis. 3rd edn. South Melbourne: MacMillan Education Australia, 2000.

27. Cui J, Huang S, Ramey G. China's Healthcare Reform and Resources Redistribution: Lessons For Emerging Nations. Review of Economic and Business Studies, Alexandru loan Cuza University, Faculty of Economics and Business Administration 2009; November (4):27-42.

28. Krieger N, Fee E. Social class: the missing link in US health data. Int $J$ Health Serv 1994;24:25-44.

29. Batty GD, Der G, Macintyre S, et al. Does IQ explain socioeconomic inequalities in health? Evidence from a population based cohort study in the west of Scotland. BMJ 2006;332:580-4.

30. Lum T. Long-term care in Asia. J Gerontol Soc Work 2012;55:563-9.

31. Australian Bureau of Statistics (ABS). Older people in cared accommodation' in Australian Social Trends 2006. Cat. no. 41020, Canberra, 2006. http://www.abs.gov.au/ausstats/abs@.nsf/Lookup/ 2071.0main+features602012-2013\#backEndnote4

32. Anderson RT, Sorlie P, Backlund E, et al. Mortality effects of community socioeconomic status. Epidemiology 1997;8:42-7.

33. Woodward M, Brindle P, Tunstall-Pedoe $\mathrm{H}$. Adding social deprivation and family history to cardiovascular risk assessment: the ASSIGN score from the Scottish Heart Health Extended Cohort (SHHEC). Heart 2007;93:172-6.

34. Mackenbach JP, Huisman M, Andersen $\mathrm{O}$, et al. Inequalities in lung cancer mortality by the educational level in 10 European populations. Eur J Cancer 2004;40:126-35.

35. Menvielle G, Kunst AE, Stirbu I, et al. Educational differences in cancer mortality among women and men: a gender pattern that differs across Europe. Br J Cancer 2008;98:1012-19.

36. Yost $\mathrm{K}$, Perkins $\mathrm{C}$, Cohen $\mathrm{R}$, et al. Socioeconomic status and breast cancer incidence in California for different race/ethnic groups. Cancer Causes Control 2001;12:703-11.

37. Krieger N, Quesenberry C, Jr., Peng T, et al. Social class, race/ ethnicity, and incidence of breast, cervix, colon, lung, and prostate cancer among Asian, Black, Hispanic, and White residents of the San Francisco Bay Area, 1988-92 (United States). Cancer Causes Control 1999;10:525-37.

38. Woodward M. Small area statistics as markers for personal socia status in the Scottish heart health study. J Epidemiol Community Health 1996;50:570-6.

39. Barzi F, Woodward M. Imputations of missing values in practice: results from imputations of serum cholesterol in 28 cohort studies. Am J Epidemiol 2004;160:34-45. 